\title{
FLAWS IN BLACK HOLE THEORY AND GENERAL RELATIVITY
}

\author{
STEPHEN J. CROTHERS \\ Alpha Institute for Advanced Study \\ PO Box 1546, Sunshine Plaza, 4558, QLD, Australia \\ *E-mail: thenarmis@gmail.com \\ www.sjcrothers.plasmaresources.com/index.html
}

\begin{abstract}
All alleged black hole models pertain to a universe that is spatially infinite, is eternal, contains only one mass, is not expanding, and is asymptotically flat or asymptotically not flat. But the alleged big bang cosmology pertains to a universe that is spatially finite (one case) or spatially infinite (two different cases), is of finite age, contains radiation and many masses including multiple black holes (some of which are primordial), is expanding, and is not asymptotically anything. Thus the black hole and the big bang contradict one another - they are mutually exclusive. It is surprisingly easy to prove that neither General Relativity nor Newton's theory predicts the black hole. Despite numerous claims for discovery of black holes in their millions, nobody has ever actually found one. It is also not difficult to prove that General Relativity violates the usual conservation of energy and momentum. Fundamentally there are contradictions contained in black hole theory, big bang cosmology, and General Relativity. Numerical methods are therefore to no avail.

Keywords: Black Hole, Big Bang, Superposition
\end{abstract}

\section{INTRODUCTION}

General Relativity has long been the theoretical basis for gravitation and the structure of the Universe. It is used to predict and characterise black holes, although it is also frequently claimed that Newton's theory predicts black holes as well. There is now a vast literature on the theory and discovery of black holes and their features, despite the fact that nobody has ever found one. It is not difficult to prove that the black hole and big bang cosmology actually contradict one another; in other words they are mutually exclusive.

\section{BLACK HOLE AND BIG BANG CONTRADICTORY}

All alleged solutions to Einstein's field equations for the black hole pertain to a universe that is spatially infinite, is eternal, contains only one mass, is not expanding, and is asymptotically flat or asymptotically not flat (e.g. are asymptotically de Sitter or anti de Sitter space). But the alleged big bang models pertain to a universe that is spatially finite (one case) or spatially infinite (two different cases), of finite age, contains radiation and many masses including multiple black holes (some of which are said to be primordial), is expanding, and is not asymptotically 
anything. Thus the black hole and big bang cosmology contradict one another; they are mutually exclusive. Furthermore, Einstein's field equations are nonlinear and so the Principle of Superposition does not hold in General Relativity; but it does hold in Newton's theory.

"The Einstein equations are nonlinear. Therefore for gravitational fields the principle of superposition is not valid." Landau and Lifshitz ${ }^{1}$

Thus, if $\mathbf{X}$ and $\mathbf{Y}$ are two different solutions to Einstein's field equations then the linear combination $a \mathbf{X}+b \mathbf{Y}$, where $a$ and $b$ are scalars, is not a solution. Physically this means that one cannot simply pile up masses and radiation in any given spacetime to obtain multiple masses and radiation. Additionally, there are no known solutions to Einstein's field equations for two or more masses and there is no existence theorem by which it can even be asserted that the field equations contain latent solutions for two or more masses. ${ }^{2-6}$ Consequently it is not possible to insert a black hole universe into a big bang universe or into another black hole universe, or to insert a big bang universe into a black hole universe or another big bang universe. Nonetheless astrophysical scientists routinely and incorrectly claim the existence of multiple black holes and the formation of black holes from objects such as stars by means of irresistible gravitational collapse.

\section{THE SCHWARZSCHILD RADIUS IS NEITHER A RADIUS NOR A DISTANCE}

The Schwarzschild radius, sometimes called the gravitational radius, features prominently in black hole theory. This radius is just that of the so-called event horizon of the black hole. Consider Hilbert's solution ${ }^{7,8}$ usually given as,

$$
\begin{gathered}
d s^{2}=\left(1-\frac{2 m}{r}\right) d t^{2}-\left(1-\frac{2 m}{r}\right)^{-1} d r^{2}-r^{2}\left(d \theta^{2}+\sin ^{2} \theta d \varphi^{2}\right) \\
0 \leq r
\end{gathered}
$$

According to Penrose, ${ }^{9}$

$$
\text { "The quantity } m \text { is the mass of the body..." }
$$

Schwarzschild's ${ }^{10}$ actual solution is different to Hilbert's and contains no black hole. In expression (1) the speed of light $c$ and Newton's gravitational constant $G$ are both set equal to unity. This practice is misleading and so with $c$ and $G$ written explicitly so that nothing is hidden, expression (1) becomes,

$$
\begin{gathered}
d s^{2}=\left(1-\frac{2 G m}{c^{2} r}\right) d t^{2}-\left(1-\frac{2 G m}{c^{2} r}\right)^{-1} d r^{2}-r^{2}\left(d \theta^{2}+\sin ^{2} \theta d \varphi^{2}\right) \\
0 \leq r
\end{gathered}
$$


The quantity $r$ in Hilbert's solution has never been correctly identified by astrophysical scientists. It has been variously and vaguely called a distance, the radius, the radius of a 2-sphere, the coordinate radius, the radial coordinate, the radial space coordinate, the areal radius, the reduced circumference, the Schwarzschild rcoordinate, the shortest distance a ray of light must travel to reach the centre, and even a gauge choice: it defines the coordinate $r$. In the particular case of $r=2 \mathrm{Gm} / \mathrm{c}^{2}$ it is invariably referred to by proponents of the black hole as the Schwarzschild radius or the gravitational radius. Dirac ${ }^{11}$ calls $r=2 m$ "the critical radius" and also says, "It would seem that $r=2 m$ gives a minimum radius for a body of mass $m$." Penrose ${ }^{9}$ says, "The radius $r=2 m$ is referred to as the Schwarzschild radius of the body." To correctly identify the quantity $r$ consider the First Fundamental Quadratic Form ${ }^{3,4,12}$ for the surface in Hilbert's solution,

$$
d s^{2}=r^{2}\left(d \theta^{2}+\sin ^{2} \theta d \varphi^{2}\right)
$$

One of the most important geometric features of a surface is its Gaussian curvature $K$, which is an intrinsic property of a surface ${ }^{\mathrm{a}}$. For a two dimensional surface this can be calculated by,

$$
K=\frac{R_{1212}}{g}
$$

$R_{1212}$ is a component of the Riemann tensor. Applying (4) to (3) gives,

$$
K=\frac{1}{r^{2}} \quad r=\frac{1}{\sqrt{K}}
$$

This now fully determines $r$ as the inverse square root of the Gaussian curvature of the spherically symmetric geodesic surface in the spatial section of Hilbert's metric, and so it is neither a radius nor a distance therein. Consequently the Schwarzschild radius is the radius of nothing in Hilbert's solution; it is not even a distance therein. The radius $R_{p}$ in the spatial section of Hilbert's solution is given elsewhere. ${ }^{3,4,13}$

Consider the first two components of the metric tensor of expression (2),

$$
g_{00}=\left(1-\frac{2 G m}{c^{2} r}\right) \quad g_{11}=-\left(1-\frac{2 G m}{c^{2} r}\right)^{-1}
$$

When $r=2 G m / c^{2}$ it is routinely asserted (e.g. Dirac ${ }^{11}$ ) that,

$$
g_{00}=(1-1)=0 \quad g_{11}=\frac{-1}{(1-1)}=\frac{-1}{0}=-\infty
$$

and that a trapped surface is produced in the course of gravitational collapse, ${ }^{2,15}$ and that the quantity $r=2 G m / c^{2}$ is the Schwarzschild radius of the black hole. However, in expressions (7) there is division by zero in the case of $g_{11}$; which is undefined in mathematics. It is also noted that not only is division by zero permitted to generate the Schwarzschild radius for the event horizon of the black hole, division

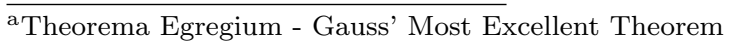


by zero is also alleged to produce $-\infty$. This too is incorrect. Since $g_{11}$ is undefined at $r=2 G m / c^{2}$ expression (2) is undefined at this value, and so no physical entity can be assigned to this value of $r$. In the case of $r=0$ expressions (6) give,

$$
g_{00}=\left(1-\frac{2 G m}{0}\right) \quad g_{11}=\frac{-1}{\left(1-\frac{2 G m}{0}\right)}
$$

Once again division by zero results, not once but twice. In this case both $g_{00}$ and $g_{11}$ are undefined and so expressions (1) and (2) are undefined. Nonetheless, the proponents of the black hole again permit division by zero and assign to this value of $r$ an infinitely dense point-mass singularity.

"Once a body of matter, of any mass $m$, lies inside its Schwarzschild radius $2 m$ it undergoes gravitational collapse . . . and the singularity becomes physical, not a limiting fiction." Dodson and Poston ${ }^{14}$

\section{THE BLACK HOLE HAS NO ESCAPE VELOCITY}

Consider the expression for the Schwarzschild radius of the black hole event horizon,

$$
r=\frac{2 G m}{c^{2}}
$$

Solving for $c$ gives,

$$
c=\sqrt{\frac{2 G m}{r}}
$$

which is Newton's escape velocity. In the Collins Encyclopaedia of the Universe, ${ }^{15}$

"black hole A massive object so dense that no light or any other radiation can escape from it; its escape velocity exceeds the speed of light."

But it is also claimed that nothing can even leave the black hole. Hawking ${ }^{16}$ says,

"I had already discussed with Roger Penrose the idea of defining a black hole as a set of events from which it is not possible to escape to a large distance. It means that the boundary of the black hole, the event horizon, is formed by rays of light that just fail to get away from the black hole. Instead, they stay forever hovering on the edge of the black hole."

Thus, the black hole is alleged to have an escape velocity and to have no escape velocity at one and the same time. Contra hype! Furthermore, if the black hole has an escape velocity $c$, then, by definition, light can escape. If the escape velocity of the black hole is greater than $c$ then light cannot escape, but that does not mean that nothing can leave, only that nothing can escape. The idea of black hole escape velocity is just a play on the words "escape velocity". ${ }^{2}$ This fact also invalidates the Hawking-Penrose Singularity Theorem. ${ }^{17}$

Equations (9) and (10) have nothing to do with the black hole whatsoever; they are related only to Newton's theory of gravitation, and equation (9) is the critical 
radius for the formation of the theoretical Michell-Laplace dark body, which is not a black hole. The theoretical Michell-Laplace dark body forms when,

$$
r<\frac{2 G m}{c^{2}}
$$

\section{NEWTON'S THEORY DOES NOT PREDICT THE BLACK HOLE}

It is incorrectly claimed that the Michell-Laplace dark body is a black hole. Hawking and Ellis ${ }^{18}$ say,

"Laplace essentially predicted the black hole..."

Chandrasekhar ${ }^{19}$ says,

"By a curious coincidence, the limit $R_{s}$ discovered by Laplace is exactly the same that general relativity gives for the occurrence of the trapped surface around a spherical mass."

Chandrasekhars' $R_{s}$ is the Schwarzschild radius. But it is not surprising that General Relativity gives the same $R_{s}$ "discovered by Laplace" because the Newtonian expression for escape velocity is deliberately inserted post hoc into Hilbert's solution in order to arbitrarily make a massive source appear therein.

The Michell-Laplace dark body possesses an escape velocity, but the black hole has no escape velocity; masses and light can leave the Michell-Laplace dark body, but nothing can leave the black hole; it does not require irresistible gravitational collapse, whereas the black hole does; it has no infinitely dense singularity, but the black hole does; it has no event horizon, but the black hole does; there is always a class of observers that can see the Michell-Laplace dark body, but there is no class of observers that can see the black hole; ${ }^{2}$ the Michell-Laplace dark body persists in a space which by consistent theory contains other Michell-Laplace dark bodies and other matter and they can interact with themselves and other matter, but the spacetime of all types of black hole pertain to a universe that contains only one mass and so cannot interact with any other masses; the space of the Michell-Laplace dark body is 3-dimensional and Euclidean, but the black hole is in a 4-dimensional nonEuclidean spacetime; the space of the Michell-Laplace dark body is flat whereas the curved spacetime of the black hole is asymptotically flat or asymptotically curved. Therefore, the Michell-Laplace dark body does not possess the characteristics of the black hole and so it is not a black hole.

\section{Ric $=0$ CONTAINS NO MATTER}

Einstein's field equations,

"couple the gravitational field (contained in the curvature of spacetime) with its sources." Foster and Nightingale ${ }^{20}$ 
Qualitatively Einstein's field equations are:

$$
\text { spacetime geometry }=-\kappa \times \text { material sources }
$$

Mathematically this is expressed as,

$$
G_{\mu \nu}=-\kappa T_{\mu \nu}
$$

where

$$
G_{\mu \nu}=R_{\mu \nu}-\frac{1}{2} R g_{\mu \nu}
$$

The energy-momentum tensor $T_{\mu \nu}$ describes the material sources of Einstein's gravitational field and the Einstein tensor $G_{\mu \nu}$ describes the curved spacetime geometry (the gravitational field) induced by the presence of the material sources. According to Einstein ${ }^{21}$ if $T_{\mu \nu}=0$ then $R=0$ and expression (11) reduces to,

$$
\text { Ric }=R_{\mu \nu}=0
$$

i.e. spacetime geometry $=0$

and he says that this describes "The Field Equations of Gravitation in the Absence of Matter". Nonetheless, Einstein and the proponents of the black hole claim that a material source is still present despite its removal. So on the one hand all material sources are removed by setting $T_{\mu \nu}=0$ then on the other hand it is claimed that a source is still present by asserting simultaneously that $R_{\mu \nu}=0$ describes the gravitational field outside a mass or "for the space between the planets in the solar system", ${ }^{11}$ or "exterior to a spherically symmetrical body", 9 and that Hilbert's solution for $R_{\mu \nu}=0$ describes the field outside a body such as a star. In relation to Hilbert's solution (1) above, Einstein ${ }^{22}$ says $^{\mathrm{b}}$,

" $M$ denotes the sun's mass, centrally symmetrically placed about the origin of co-ordinates; the solution (109a) is valid only outside of this mass, where all the $T_{\mu \nu}$ vanish. If the motion of the planet takes place in the $x_{1}-x_{2}$ plane then we must replace (109a) by

$$
d s^{2}=\left(1-\frac{A}{r}\right) d t^{2}-\frac{d r^{2}}{\left(1-\frac{A}{r}\right)}-r^{2} d \varphi^{2} "
$$

wherein $A=\kappa M / 4 \pi$. Of Hilbert's solution Dirac ${ }^{11}$ says,

"It is known as the Schwarzschild solution. It holds outside the surface of the body producing the field, where there is no matter. Thus it holds fairly accurately outside the surface of a star."

"The solution leads to small corrections in the Newtonian theory for the motions of planets around the Sun."

Penrose $^{9}$ says in relation to Hilbert's solution,

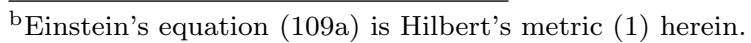


"It is known as the Schwarzschild solution. It holds outside the surface of the body producing the field, where there is no matter. Thus it holds fairly accurately outside the surface of a star."

Thus the material source is removed on the one hand by setting $T_{\mu \nu}=0$ and on the other hand immediately reinstated with the misleading words "outside this mass" or "outside the surface of a star". Einstein's argument violates elementary logic and is therefore false; nothing but a subtle circular play on the words "outside this mass". $R_{\mu \nu}=0$ actually contains no matter and so it is physically meaningless. Consequently, Hilbert's solution has no physical meaning either. Hence, the black hole is not predicted by General Relativity.

Furthermore, both Einstein and Dirac for example, have not only introduced a material source where there is none by means of the words "outside of this mass" and "the body producing the field", they have also arbitrarily and in violation of equations (12), introduced multiple masses by means of the words "motion of the planet" and "the motions of the planets". These additional planetary masses have no theoretical basis either because they too are inserted linguistically and post hoc into equations that by mathematical construction contain no matter, and where the Principle of Superposition does not hold.

\section{THE BLACK HOLE VIOLATES THE PHYSICAL PRINCIPLES OF GENERAL RELATIVITY}

Einstein $^{22}$ asserted that his Principle of Equivalence and his laws of Special Relativity must hold in sufficiently small finite regions of his gravitational field, and that these regions can be located anywhere in his gravitational field.

"Stated more exactly, there are finite regions, where, with respect to a suitably chosen space of reference, material particles move freely without acceleration, and in which the laws of special relativity, which have been developed above, hold with remarkable accuracy." Einstein ${ }^{22}$

Both the Principle of Equivalence and Special Relativity are defined in terms of the $a$ priori presence of multiple arbitrarily large finite masses and photons. It is therefore impossible for the Principle of Equivalence and Special Relativity to manifest in the spacetime $R_{\mu \nu}=0$ which by mathematical construction contains no matter. Thus $R_{\mu \nu}=0$ violates the physical principles of General Relativity. Hilbert's solution therefore is also of no physical significance. But it is from Hilbert's solution that the black hole was first obtained. It is also impossible for the Principle of Equivalence and Special Relativity to manifest in a universe that, allegedly, contains only one mass.

\section{INVALIDITY OF BIG BANG COSMOLOGY}

Big bang cosmology, after the initial bang, treats the Universe as being entirely filled by a single continuous indivisible homogeneous distribution of matter of uniform 
macroscopic density and pressure. Indeed, Tolman ${ }^{23}$ remarks,

"In interpreting the expressions for density $\rho_{00}$ and pressure $p_{0} \ldots$, it must be remembered that these quantities apply to the idealized fluid in the model, which we have substituted in place of matter and radiation actually present in the real universe." ... "Hence, although we shall actually make great use of homogenous models in our studies, we shall have to realise that we do this primarily in order to secure a definite and relatively simple mathematical problem, rather than to secure a correspondence with known reality."

Since the big bang cosmological models consist of an indivisible continuous distribution of matter none can incorporate the formation of numerous galaxies and stars and permeating radiation, by definition, and therefore do not "secure a correspondence with known reality." Multiple masses and radiation are obtained from big bang models in the very same way as multiple black holes; by invalidly applying the Principle of Superposition by means of a false analogy with Newton's theory. Tolman, ${ }^{23}$ for example, incorrectly asserts,

"We can then treat the universe as filled with a continuous distribution of fluid of proper macroscopic density $\rho_{00}$ and pressure $p_{0}$, and shall feel justified in making this simplification since our interest lies in obtaining a general framework for the behaviour of the universe as a whole, on which the details of local occurrences could later be superposed."

Furthermore, since the big bang models treat the Universe as being filled by a single indivisible mass distribution, neither the Principle of Equivalence not Special Relativity can manifest. Hence, the big band models also violate the physical principles of General Relativity in the same way as does the black hole.

The big bang singularity is also claimed to have no extension yet also an infinite density, infinite pressure and infinite temperature. According to Hawking, ${ }^{24}$

"At the big bang itself, the universe is thought to have had zero size, and to have been infinitely hot."

According to Misner, Thorne and Wheeler, ${ }^{25}$

"One crucial assumption underlies the standard hot big-bang model: that the universe 'began' in a state of rapid expansion from a very nearly homogeneous, isotropic condition of infinite (or near infinite) density and pressure."

How close to infinite must one get to be "near infinite"?

\section{INVALIDITY OF EINSTEIN'S FIELD EQUATIONS}

Einstein's field equations can be written in mixed tensor form, thus

$$
G_{\nu}^{\mu}=-\kappa T_{\nu}^{\mu}
$$


Einstein ${ }^{21}$ says that the total energy-momentum $E$ of his gravitational field and its sources is,

$$
E=\left(t_{\mu}^{\sigma}+T_{\mu}^{\sigma}\right)
$$

where $t_{\mu}^{\sigma}$ is his pseudotensor and $T_{\mu}^{\sigma}$ is his energy-momentum tensor. According to Einstein $^{21}$ the components of his pseudotensor are the

$$
\text { "energy components ... of the gravitational field." }
$$

Note that expression (14) is not a tensor sum because the pseudotensor is not a tensor, and so Einstein ${ }^{21}$ takes an ordinary divergence of expression (14), not a tensor divergence, thus,

$$
\frac{\partial\left(t_{\mu}^{\sigma}+T_{\mu}^{\sigma}\right)}{\partial x_{\sigma}}=0
$$

Einstein $^{21}$ says of equation (15),

"Thus it results from our field equations of gravitation that the laws of conservation of momentum and energy are satisfied."

However, Einstein's pseudotensor is a meaningless collection of mathematical symbols and so it cannot be used to make any calculations or to model any phenomena. Thus, Einstein's expressions (14) and (15) are both physically and mathematically untenable. Here is the proof. ${ }^{26}$ Einstein's pseudotensor is defined as, ${ }^{26}$

$$
\sqrt{-g} t_{\nu}^{\mu}=\frac{1}{2}\left[\delta_{\nu}^{\mu} L-\left(\frac{\partial L}{\partial g_{, \mu}^{s b}}\right) g_{, \nu}^{s b}\right]
$$

wherein $g$ is the determinant of the metric tensor, $\delta_{\nu}^{\mu}$ is the Kronecker-Delta symbol which has the property that $\delta_{\nu}^{\mu}=1$ when $\mu=\nu$ and is 0 otherwise, and

$$
L=-g^{\alpha \beta}\left(\Gamma_{\alpha \kappa}^{\gamma} \Gamma_{\beta \gamma}^{\kappa}-\Gamma_{\alpha \beta}^{\gamma} \Gamma_{\gamma \kappa}^{\kappa}\right) \quad \Gamma_{b c}^{a}=\frac{1}{2} g^{a d}\left(\frac{\partial g_{d c}}{\partial x^{b}}+\frac{\partial g_{b d}}{\partial x^{c}}-\frac{\partial g_{b c}}{\partial x^{d}}\right) \quad g_{, \nu}^{s b}=\frac{\partial g^{s b}}{\partial x^{\nu}}
$$

Note that $\Gamma_{b c}^{a}$ is composed solely of the components of the metric tensor $g^{c d}$ and $g_{a b}$ and their first derivatives $\partial g_{\mu \nu} / \partial x^{\sigma}$. Consequently $L$ is composed solely of the components of the metric tensor and their first derivatives. Since $g$ is the determinant of the metric tensor it is composed solely of the components of the metric tensor. Contracting Einstein's pseudotensor gives,

$$
\sqrt{-g} t_{\mu}^{\mu}=\frac{1}{2}\left[4 L-\left(\frac{\partial L}{\partial g_{, \mu}^{s b}} g_{, \mu}^{s b}\right)\right]
$$

where $t_{\mu}^{\mu}=t$, which is an invariant. Furthermore, ${ }^{26}$

$$
\left(\frac{\partial L}{\partial g_{, \mu}^{s b}}\right) g_{, \mu}^{s b}=2 L
$$

Substituting this into expression (17) and solving for $t$ gives the invariant,

$$
t=\frac{L}{\sqrt{-g}}
$$


Owing to the definitions of $L$ and $g, t$ is necessarily an invariant that is composed solely of the components of the metric tensor and their first derivatives. But the pure mathematicians G. Ricci-Curbastro and T. Levi-Civita, ${ }^{27}$ inventors of the tensor calculus, proved in 1900 that such invariants do not exist. Thus Einstein's pseudotensor is meaningless. Taking this fact into account, the fact that $R_{\mu \nu}=0$ is not meaningful, and rightly considering the conservation of energy and momentum, Einstein's field equations must take the following form, ${ }^{3-5,26,28}$

$$
\frac{G_{\nu}^{\mu}}{\kappa}+T_{\nu}^{\mu}=0
$$

Rewrite expression (14) as follows,

$$
\left(t_{\nu}^{\mu}+T_{\nu}^{\mu}\right)=E
$$

Compare expression (18) to expression (19). It is now clear that not only is expression (18) the correct form of Einstein's field equations but it is also the expression for the total energy-momentum of his gravitational field. It is also noted from expressions (18) that the $G_{\nu}^{\mu} / \kappa$ are the components of a gravitational energy tensor, ${ }^{26}$ not the $t_{\nu}^{\mu}$. Then according to expression (18) the total energy of Einstein's gravitational field is always zero. This means that the $G_{\nu}^{\mu} / \kappa$ and the $T_{\nu}^{\mu}$ must vanish identically, i.e. when $T_{\nu}^{\mu}=0$ then $G_{\nu}^{\mu}=0$ also, to yield the identity $0=0$, so that when $T_{\nu}^{\mu}=0$ there is no matter and no spacetime (i.e. no universe) and hence no gravitational field, and so Einstein's claim that his field equations reduce to $R_{\mu \nu}=0$ when $T_{\mu \nu}=0$ is untrue. It also means that gravitational energy cannot be localised (i.e. Einstein gravitational waves do not exist) ${ }^{26}$ and that Einstein's field equations violate the usual conservation of energy and momentum and are therefore in conflict with experiment on a deep level, rendering them invalid.

Consider Einstein's field equations with cosmological constant $\Lambda$ included. In view of expression (18) the field equations must be expanded to the following:

$$
\frac{\left(G_{\mu \nu}+\Lambda g_{\mu \nu}\right)}{\kappa}+T_{\mu \nu}=0
$$

where $\left(G_{\mu \nu}+\Lambda g_{\mu \nu}\right) / \kappa$ are now the components of a gravitational energy tensor, so that $\left(G_{\mu \nu}+\Lambda g_{\mu \nu}\right) / \kappa$ and $T_{\mu \nu}$ must vanish identically, with all the consequences related to expression (18). So the cosmological constant also has no physical meaning.

\section{DEDICATION}

I dedicate this paper to my late beloved brother:

\section{Paul Raymond Crothers}

12th May 1968 - 25th December 2008 


\section{References}

1. Landau, L. and Lifshitz, E., The Classical Theory of Fields, Addison-Wesley, Reading, MA, (1951)

2. McVittie, G. C., Laplace's alleged "black hole", The Observatory, v.98, 272, (1978), www.sjcrothers.plasmaresources.com/McVittie.pdf

3. Crothers, S. J., "The Schwarzschild solution and its implications for waves", (2008), Conference of the German Physical Society, Munich, March 9-13, 2009, Verhandlungen der Deutsche Physikalische Gesellschaft Munich 2009: Fachverband Gravitation und Relativittstheorie, http://vixra.org/abs/1103.0051

4. Crothers, S. J. "The Black Hole, the Big Bang: a Cosmology in Crisis", (May 2010), http://vixra.org/pdf/1103.0047v1.pdf

5. Crothers, S. J., "General Relativity - A Theory in Crisis", Global Journal of Science Frontier Research Physics and Space Science, Volume 12, Issue 4, Version 1, (June 2012), http://vixra.org/pdf/1207.0018v2.pdf

6. Crothers, S. J., "Proof of No 'Black Hole' Binary in Nova Scorpii", Global Journal of Science Frontier Research Physics and Space Science, Volume 12, Issue 4, Version 1, (June 2012), http://vixra.org/pdf/1206.0080v2.pdf

7. Abrams, L. S. "Black Holes: The Legacy of Hilbert's Error", Can. J. Phys. 67: 919, (1989), http://arXiv:gr-qc/0102055

8. Antoci, S., "David Hilbert and the origin of the 'Schwarzschild' Solution" (2001), http://arxiv.org/pdf/physics/0310104

9. Penrose, R., "Gravitational Collapse: The role of General Relativity", General Relativity and Gravitation, Vol. 34, No. 7, July (2002)

10. Schwarzschild, K., "On the gravitational field of a mass point according to Einstein's theory", Sitzungsber. Preuss. Akad. Wiss., Phys. Math. Kl., 189, (1916), www.sjcrothers.plasmaresources.com/schwarzschild.pdf

11. Dirac, P.A.M., General Theory of Relativity, Princeton Landmarks in Physics Series, Princeton University Press, Princeton, NJ, (1996)

12. Levi-Civita T. The Absolute Differential Calculus, Dover Publications Inc., New York, (1977)

13. Crothers, S. J., "On the General Solution to Einstein's Vacuum Field and its Implications for Relativistic Degeneracy", Progress in Physics, Vol. 1, 68-73, (2005), www.pteponline.com/index_files/2005/PP-01-09.PDF

14. Dodson, C. T. J., and Poston, T., Tensor Geometry - The Geometric Viewpoint and its Uses, 2nd Ed., Springer-Verlag, (1991)

15. Collins Encyclopaedia of the Universe, Harper Collins Publishers, London, (2001)

16. Hawking, S. W., The Theory of Everything, The Origin and Fate of the Universe, New Millennium Press, Beverly Hills, CA, (2002)

17. Crothers, S. J., "On the Invalidity of the Hawking-Penrose Singularity 'Theorems' and Acceleration of the Universe with Negative Cosmological Constant", Global Journal of Science Frontier Research Physics and Space Science, Volume 13 Issue 4, Version 1.0, June 2013, http://vixra.org/abs/1304.0037

18. Hawking, S. W. and Ellis, G. F. R., The Large Scale Structure of Space-Time, Cambridge University Press, Cambridge, (1973)

19. Chandrasekhar, S., "The increasing role of general relativity in astronomy", The Observatory, 92, 168, (1972)

20. Foster, J. and Nightingale, J. D., A short course in General Relativity, Springer-Verlag, New York, Inc., (1995)

21. Einstein, A. The Foundation of the General Theory of Relativity, Annalen der Physik, 49, (1916), The Principle of Relativity (A collection of original memoirs on the special and general theory of relativity), Dover Publications Inc., New York, (1952)

22. Einstein, A. The Meaning of Relativity, Science Paperbacks and Methuen \& Co. Ltd., (1967)

23. Tolman, R. C., Relativity Thermodynamics and Cosmology, Dover Publications Inc., New York, (1987)

24. Hawking, S. W., A Brief History of Time from the Big Bang to Black Holes, Transworld Publishers Ltd., London, (1988) 
25. Misner C. W., Thorne K. S., Wheeler J. A., Gravitation, W. H. Freeman and Company, New York, (1970)

26. Levi-Civita, T., "Mechanics. - On the Analytical Expression that Must be Given to the Gravitational Tensor in Einstein's Theory", Rendiconti della Reale Accadmeia dei Lincei 26: 381 (1917), http://arxiv.org/pdf/physics/9906004

27. Ricci-Curbastro, G., Levi-Civita, T., Méthodes de calcul différential absolu et leurs applications, Matematische Annalen, B. 54, 1900, p.162.

28. Lorentz, H. A., Versi. Gewone Vergad. Akad., 24, 1389 and 1759, (1916); 25, 468 and 1380 , (1916) 